\title{
Effectiveness of Cardiac Scintigraphy and Standard Eco-Cardiograph in the Detection and Evaluation of Cardiac Disorders, Including Ischemic Heart Diseases
}

\author{
Saeed M. Bafaraj \\ Diagnostic Radiology Department, Faculty of Applied Medical Sciences, King Abdulaziz University, Jeddah, KSA \\ Email: smbafaraj@kau.edu.sa
}

How to cite this paper: Bafaraj, S.M. (2021) Effectiveness of Cardiac Scintigraphy and Standard Eco-Cardiograph in the Detection and Evaluation of Cardiac Disorders, Including Ischemic Heart Diseases. Journal of Biosciences and Medicines, 9 , 90-109.

https://doi.org/10.4236/jbm.2021.94007

Received: January 23, 2021

Accepted: April 16, 2021

Published: April 19, 2021

Copyright $\odot 2021$ by author(s) and Scientific Research Publishing Inc. This work is licensed under the Creative Commons Attribution International License (CC BY 4.0). http://creativecommons.org/licenses/by/4.0/

\section{Open Access}

\begin{abstract}
Background: Cardiac disorders are the leading causes of morbidity and mortality globally. Aim: The current study aimed to compare the effectiveness of echocardiographic and scintigraphy investigation in cardiovascular disorders detection and evaluation, including ischemic heart disease (IHD) diagnosis at King Abdulaziz University in Saudi Arabia. Methods: 157 patients (male/ female: $68 \% / 32 \%$; mean age $64 \pm 0.83$ years) were included in the report in the current study. All subjects underwent gated myocardial perfusion scintigraphy and standard Echocardiography. Results: About $84 \%$ of cases were diagnosed with any types of cardiac disorders by Echocardiography, whereas through scintigraphy, $61 \%$ of patients were detected cardiac disorders. The age stratification did not impact the prevalence of cardiac disorders detected by them. Scintigraphy showed a higher detection rate (59\%), while only $29 \%$ of cases were detected with IHD by eco-cardiograph. Conclusions: In conclusion, both techniques, namely standard Echocardiography, and myocardial scintigraphy, are useful in the evaluation and detection of cardiac disorders in patients having any type of cardiac problems. Both investigations showed a differential pattern in cardiac disorders diagnosis with a particular focus on IHD. Gender differences and age stratification also contributed to this differential pattern of diagnosis. Trial Registration: The research doesn't include experiments in humans or animals. It is a retrospective study for data record review as an observational study, so no trial registration is required.
\end{abstract}

\section{Keywords}

Cardiac Disorders, Evaluation, Comparison, Standard Echocardiography, Cardiac Scintigraphy 


\section{Introduction}

Cardiovascular diseases are the most common cause of disability and mortality worldwide, with a prevalence of 126 million persons affected globally (1655 per 100,000) and 17.9 million mortalities making it $31 \%$ of all global deaths [1] [2]. The term "heart disease or cardiac disorders" refers to several conditions where heart functions or structures are impaired and abnormal [3]. Characterized by atherosclerosis in the arteries, coronary artery disease (CAD) is the most common heart disease which is also the most frequent cause of heart attack [2]. Other significant cardiac disorders are valvular disorders, congenital heart diseases, and others. More than 600,000 Americans die from cardiac or heart disease every year, leading to one in every four deaths at the statistical level. Though the efforts of prevention of cardiovascular disease at primary, secondary, and tertiary levels are mandatory, diagnostic modalities at this regard need to be re-visited and re-evaluated from various contexts. A lot of arrangements should be and can be made at different stages of health care services to combat this life killer through early diagnosis and consequent therapeutic and preventive strategies.

CAD is regarded as the most common fatal disease all over the world [4]. The identification of patients with myocardial ischemia is an integral part of the diagnosis of CAD [1] [2]. To date, Echocardiography is a first-line investigation to detect cardiac disorders. Echocardiography is the potential in providing in-depth and thorough comprehensive profiling of both the structural, morphological, anatomical, and hemodynamic changes which are induced and aggravated by either acute or chronic CAD [5] [6]. Thus, over a long time standard, Echocardiography occupies a vital position in evaluating the CAD in saving a hundred thousand lives. Standard Echocardiography, the most widespread investigation in cardiology, has numerous objectives in the vulnerable cardiac population, including the valuation of myocardial performance and function, valvular diseases, congenital cardiac abnormalities, hemodynamics [5] [6].

Indeed, Echocardiography, including both two-dimensional and, in recent times, three-dimensional speckle tracking Echocardiography, exhibit reliable methods for the detection and assessment of global as well as regional myocardial dysfunction has as often seen various cardiovascular diseases [7] [8]. Thus, these unique and innovative echocardiographic imaging modalities have broadened the knowledge of LV and RV mechanics, physiology, pathology, and morphology. A striking drawback of this study's valuation and interpretation is the subjective visual analysis of endocardial motion and wall thickening that is only semiquantitative needing more quantitative techniques. Indeed, further advancement has already taken place to the incorporation of new indices in the analysis of regional wall motion.

On the other hand, myocardial perfusion scintigraphy (MPS) is regarded as an accurate and sensitive modality in diagnosing the ischemia affected regions in the myocardial tissue [9] [10] [11]. Myocardial perfusion single-photon emission 
computed tomography is considered to be the most potential and commonly performed cardiac non-invasive imaging technologies. Scintigraphy is unique in playing a key and vital role in the diagnosis and detection of cardiovascular disorders, with assessing the disease prognosis, establishing therapeutic effectiveness and contributes to evaluating myocardial viability [9] [11]. In this regard, other potential diagnostics can be into consideration, such as cardiac computed tomography scan or cardiac magnetic resonance imaging [12] [13]. Despite all these advances, scintigraphy is still the most essential and sensitive modality in showing and viewing the ischemia. Indeed, scintigraphy is taken as a reference gold standard method in several recent studies in which various stress Echo protocols are introduced [14] [15].

The present study intended to compare the effectiveness and contribution of standard eco-cardiograph and nuclear medicine scintigraphy in detecting and evaluating cardiac disorders at a tertiary level hospital in Saudi Arabia. The objective of the study also includes to find their effectiveness based on gender and age of patients. This is the first effort to compare and contrast the detection of cardiac diseases in Saudi patients using two popular and standard diagnostic methodologies used in the field of cardiology. Here, the patients diagnosed having cardiac disorders without further stratification were evaluated.

\section{Aim}

The aim of the study was to compare the effectiveness of echocardiographic and scintigraphy investigation in cardiovascular disorders detection and evaluation, including ischemic heart disease (IHD) diagnosis at a tertiary level hospital in Saudi Arabia.

\section{Materials and Methods}

\subsection{Study Population}

One hundred sixty-six subjects underwent echocardiography and nuclear medicine imaging at the hospital. These patients took both Echocardiography and nuclear imaging scintigraphy. In this study, clinical data records of patients with cardiac problems were analyzed, while cancer patients were excluded. Here broadly, the patients diagnosed with cardiac disorders were included (such as with ischemia, infarction, IHD and other); there were no specific criteria for cardiac disorders patient selection in this study. Adult patients with both genders were included and were later characterized based on gender and age less than or greater than 50 years. For IHD, they needed to be identified by Echo findings.

The study protocol has been reviewed and approved by the Institutional Ethics Committee. All patients have provided written informed consent. As this study involved human participants, therefore it was in accordance with the Helsinki declaration and standard ethic standard of the Institutional Ethics Committee. Specific purpose, objective, and risks of the nuclear imaging technique have been clearly explained to study subjects. 


\subsection{Study Materials}

The patients underwent nitrate-enhanced rest scintigraphy with technetium-99mmethoxy isobutyl isonitrile (99mTc-MIBI). Technetium-99m-sestamibi is a myocardial perfusion radiotracer that has been commercially available in the United States since 1990 [16] [17] [18]. Since the recent introduction of technetium-99m-labeled radiotracers for myocardial perfusion imaging, a variety of protocols for the performance of this procedure have been developed [16] [17].

\subsection{Echocardiographic Examination}

The EC was accomplished by Vivid 7 instruments (GE Medical Systems, Milwaukee, WI, USA), with a $2.5-\mathrm{MHz}$ transducer and harmonic imaging. Echocardiographic assessments were done on the patients with lying in the left lateral decubitus position under the recommendations and protocols of the American Society of Echocardiography [19]. Two-dimensional images were captured at the apical four- and five-chambers and the long axis. Indeed, both the apical four-chamber and two-chamber views are regarded as necessary in Echocardiography to assess wall motion disorders. The left ventricular systolic and diastolic diameters (LVS, LVD) were obtained by M-mode echocardiography. The LV-ejection fraction (LV-EF) was determined by applying the altered version of biplane Simpson's method.

Strain (S) and strain rate (SR) were tracked by the speckle tracking. Tissue Doppler-derived LVS longitudinal S and SR rates were attained in two- and four-chamber apical views. Through Echocardiography 16 segments were assessed, namely basal, middle, and apical parts of the septum, inferior, lateral, and anterior walls, and basal and middle segments of the anterior septum and posterior wall. Recordings were taken at the end of the expiration phase continuing three consecutive cycles. The well-trained specialized doctors and supporting staffs have conducted the echocardiographic assessment.

\subsection{Nuclear Medicine Scintigraphy Imaging Protocol}

The study was conducted at the Department of Nuclear Medicine by specialty medical doctors, who were wholly blinded to the other diagnostics and risk factors data. All enrolled participants had to undergo a rest protocol with 99mTc-MIBI. Patients are prohibited from having anything orally (except oral administration of medicine or water) after midnight before the examination to restrict gut activity as it may affect the assessment of the inferior wall of the LV. Patients must be dressed comfortably for the exercise part of the investigation. Medicines comprising methylxanthines or caffeine and caffeine enriched food-beverage should be kept away for at least $12-24$ hours. Metal or other potential attenuators should be taken out before imaging to prevent attenuation artifacts. Before administering 99mTc-MIB, registered patients were provided with $1-2$ tablets of sublingual nitroglycerin $(0.4 \mathrm{mg})$ with a duration of 5 min apart. Finally, 740 $\mathrm{MBq}$ of $99 \mathrm{mTc}-\mathrm{MIBI}$ was injected through the intravenous route while resting, 
and scintigraphy investigation was performed after $45 \mathrm{~min}$.

99mTcsestamibi and $99 \mathrm{mTc}$-tetrofosmin are the bulk utilized radiopharmaceuticals in cardiac scintigraphy. These radiopharmaceuticals show extraction efficiency of around $65 \%$ in the physiological flow range; the absorption is in relative equilibrium to flow. As compared to the hepatobiliary excretion of 99mTctetrofosmin, the hepatobiliary excretion of sestamibi is less, and evaluation of the inferior wall is less challenging. The conventional protocol of 201Tl-chloride comprises stress imaging and consequently redistribution imaging past 3 or 4 hours. When delayed, imaging is attained after 24 hours to assess fixed faults (primarily to evaluate the myocardium feasibility). The second 201Tl-chloride procedure includes the performance of the elective delayed images on the same day, following an injection of a little dose of 201Tl-chloride in patients with a fixed defect. The third 201Tl-chloride protocol includes acquiring the 3 or 4 -hour images followed by the injection of a small amount of 201Tl-chloride, and delayed images at 24 hours are still performed when required.

99mTc utilizes four protocols. The original protocol consists of two injections of the radiopharmaceuticals on two different days. The original procedure was altered for the convenience of patients, preferring a low dose and high dose on the same day, initiating with either the most commonly used, rest and then stress (second protocol) or, stress than rest (third protocol). In the fourth protocol, the so-called dual isotopes, 201Tl-chloride images are first taken by resting injection, followed by which and $99 \mathrm{mTc}$ radiopharmaceutical is injected during stress. Above mentioned protocols have their proponent, that highlights the logistical, practical consequences, as available scientific evidence has not established the clarity of any one of these protocols. Patients with CAD and severe LV dysfunction, can provide resting imaging to determine normal or near-normal uptake of either $99 \mathrm{mTc}$ agents or 201Tl-chloride and, consequently, myocardial viability [15].

We identified ischemic heart disease in scintigraphy by myocardial perfusion imaging as a nuclear stress test and via pharmacologic stress.

\subsection{Definition Criteria of the Positive Diagnostic Imaging Tool}

Positive stress echocardiography is defined as the presence of abnormal cardiac wall motion on either exercise or pharmacologic stress echocardiography.

A positive nuclear medicine means the heart's blood flow is insufficient, may occur only during the exercise phase of the stress test.

\subsection{Statistical Analysis}

Statistical analysis was done with SPSS 20 software package (version 17, SPSS Inc., Chicago, IL, USA). The study parameters were expressed as mean \pm standard error or as a percentage. We used the crosstabs chi-square test for categorical and independent variables. Samples T-Test was used for continuous va- 
riables.

\section{Results}

\subsection{Basic Profiles of Study Participants}

Although 166 patients were recruited for the current study, because of the presence of some missing data, 157 data for the final analysis were included (Figure $1)$.

Table 1 demonstrated the basic profiles of study participants. The mean age was $64.0 \pm 0.83$ years; the ratio of male and female participants was 107:50.

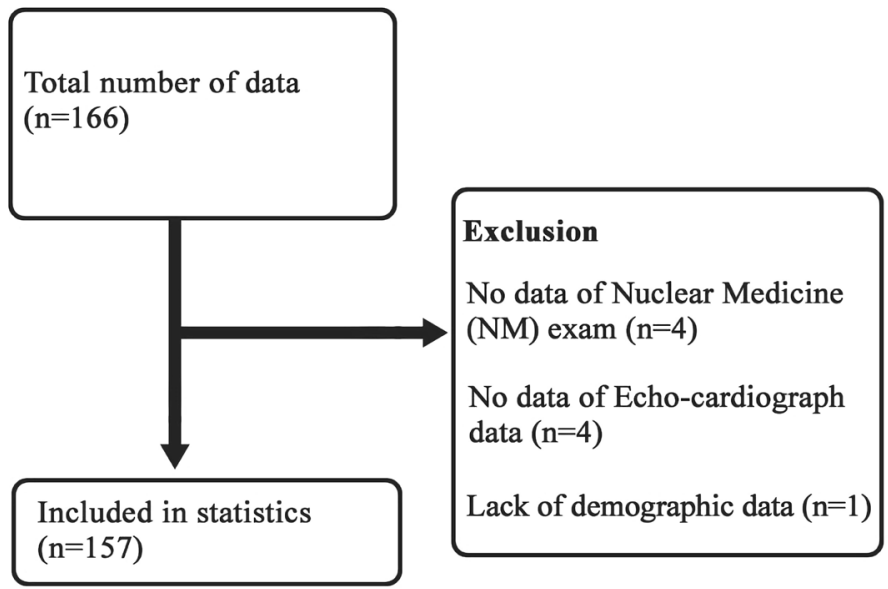

Figure 1. Participants data.

Table 1. Patients characteristics.

\begin{tabular}{ccccc}
\hline Variables & Total $(\mathbf{n}=157)$ & Male $(\mathbf{n}=107)$ & Female (n=50) & P value \\
\hline Demographics & & & & \\
Age, years & $64.0 \pm 0.83$ & $64.3 \pm 1.03$ & $63.5 \pm 1.43$ & 0.641 \\
Age $\geq 50$ years & $145(92.4)$ & $98(91.6)$ & $47(94.0)$ & 0.596 \\
Age < 50 years & $12(7.6)$ & $9(8.4)$ & $3(6.0)$ & \\
Echocardiography & & & & 0.131 \\
Echocardiography (positive) & $131(83.4)$ & $86(80.4)$ & $45(90.0)$ & 0.033 \\
IHD (identified by Echo findings) & $46(29.3)$ & $37(34.6)$ & $9(18.0)$ & 0.050 \\
Nuclear medicine & & & & 0.068 \\
Nuclear medicine (positive) & $96(61.1)$ & $71(66.4)$ & $25(50.0)$ & $13(26.0)$ \\
Ischemia & $35(22.3)$ & $22(20.6)$ & $4(8.0)$ & \\
Infarction & $30(19.1)$ & $26(24.3)$ & $7(14.0)$ & \\
Ischemia and Infarction & $29(18.5)$ & $22(20.6)$ & $2(4.0)$ & \\
Others & $6(3.8)$ & $4(3.7)$ & $24(48.0)$ & 0.038 \\
\hline IHD (identified by NM findings) & $94(59.9)$ & $70(65.4)$ &
\end{tabular}

$\mathrm{N}=157$, Values are mean \pm standard error or $\mathrm{n}(\%)$, IHD: ischemia heart diseases. Based on T-test for continuous variable and Pearson Chi-square test for the categorical variable. 
There was a $7.6 \%$ study participant who was less than 50 years old. All patients were studied at rest and stress.

Among the study patients who underwent standard echo-cardiograph, 83.4\% had been diagnosed with cardiac disorders while with scintigraphy, and this percent was $61.1 \%$ (cardiac disorder is any disorder that affects/involves the heart or its blood vessels). Here $29.3 \%$ of participants were diagnosed with IHD by eco-cardiograph. In contrast, the detection rate of IHD was higher by scintigraphy, and that was 59\%, more than two-fold higher than that of Echocardiography. The prevalence of IHD is higher in the male group than the female groups by both technologies. The prevalence of cardiac disorders in the age group more than 50 years was 10-fold higher than the age group below 50 by both diagnostics. Looking at the subtypes of IHD, a very close prevalence rate of isolated ischemia, isolated infarction, and both are found. A bit more detail of cardiac abnormalities detected by both techniques were demonstrated in Table 2(A), Table 2(B).

\subsection{Comparison by Both Technologies for Cardiac Disorders}

The various abnormalities detected in echo-cardiography and scintigraphy of current study participants have been listed in Table 3(A), Table 3(B). As shown

Table 2. (A) Cardiac disorder diagnosed by echocardiography; (B) Cardiac disorder diagnosed by nuclear medicine (scintigraphy).

(A)

\begin{tabular}{cccc}
\hline Name of cardiac disorder & Total number & Disorder number & Percentage (\%) \\
\hline Echocardiography (positive) & 157 & 131 & 83.4 \\
IHD (identified by Echo findings) & 157 & 46 & 29.3 \\
Normal global LV systolic function & 157 & 53 & 33.8 \\
Mildly decreased global LV systolic function & 157 & 9 & 4.46 \\
Moderately decreased global LV systolic function & 157 & 7 & 1.27 \\
Severely decreased global LV systolic function & 157 & 2 & 2.55 \\
Hypertensive heart disease & 157 & 4 & \\
\hline
\end{tabular}

IHD: ischemia heart diseases; LV: left ventricular.

(B)

\begin{tabular}{cccc}
\hline Name of cardiac disorder & Total number & Disorder number & Percentage (\%) \\
\hline Nuclear medicine (positive) & 157 & 96 & 61.1 \\
Ischemia & 157 & 35 & 22.3 \\
Infarction & 157 & 30 & 19.1 \\
Ischemia and Infarction & 157 & 29 & 18.5 \\
IHD (identified by NM findings) & 157 & 94 & 59.9 \\
Others & 157 & 6 & 3.8 \\
\hline
\end{tabular}

IHD: ischemia heart diseases. 
Table 3. (A) Echocardiography positive findings; (B) Nuclear medicine (scintigraphy) positive findings.

(A)

Mildly decreased LV systolic function

Impaired relaxation pattern pf LV diastolic filling

Severely dilated LA

Mild LV dysfunction

Borderline concentric LV hypertrophy

Mildly dilated LA

Moderately to severely decreased Normal global LV systolic function

Mildly increased LV internal cavity size

Sclerosed aortic valve

Mild LV systolic dysfunction

Difficult subcostal view

Mild thickening and calcification of the posterior mitral valve leaflet

Mild mitral annular calcification

Mild aortic regurgitation

Mild concentric LV hypertrophy

Trivial pericardial effusion

Mildly elevated pulmonary artery systolic pressure

Mildly to moderately decreased global LV systolic function

Multiple segmental abnormalities exist

RCA distribution is abnormal

Mild to moderately mitral valve regurgitation.

Mild-moderate tricuspid regurgitation.

Moderately dilated LA

Severe mitral valve regurgitation

Mid and apical anterior wall, basal and mid anterior septum, basal inferoseptal segment, and apex are abnormal

Positive stress echo for coronary angiography

Mild to moderate mitral valve regurgitation

Pulmonary artery systolic pressure is normal with a decrease in severity of tricuspid regurgitation

Mild mitral valve regurgitation mild tricuspid regurgitation

Mild thickening of the anterior and posterior mitral valve leaflet

Normal LV systolic function

Mild mitral stenosis

Rheumatic heart disease

Mild mitral valve regurgitation, moderate aortic regurgitation

Trivial tricuspid regurgitation with normal PASP

Moderately decreased global LV systolic function

Mild aortic valve sclerosis

Moderately enlarged RV

Moderately elevated pulmonary artery systolic pressure

Non-dilated LV with good systolic function

Trivial tricuspid regurgitation

Mildly decreased global LV systolic function

Fair LV systolic function

Difficult echo study, suboptimal echo windows

Trace mitral regurgitation, trace tricuspid regurgitation, trace pulmonary regurgitation

Mild thickening and calcification of the anterior mitral valve leaflet

Diastolic dysfunction

Trivial mitral regurgitation

Concentric LV hypertrophy

Moderate mitral valve regurgitation

Moderate LV systolic dysfunction

Mild pulmonary hypertension

Poor echo window 


\section{Continued}

Nondilated LV with overall good systolic function

Trivial aortic regurgitation

Sclerocalcific changes of mitral and aortic valve

Mild aortic stenosis

Mildly decrease global LV systolic function

Type 3 LV diastolic dysfunction

Regional wall motion abnormality

Dilated LV with severely impaired systolic function

Moderately decrease global LV systolic function

Mildly increase LV internal cavity size

Aortic valve disease with mild aortic regurgitation

Mitral valve prolapses with travail mitral regurgitation

Dilated ischemic cardiomyopathy

Impaired relaxation pattern of LV diastolic filling

Small secundum arterial septal defect with predominantly right to left across the atrial septum

Mildly dilated RA

Moderate tricuspid regurgitation

IHD, S\P CABG,

Regional wall motion abnormalities

Mild thickening aortic valve with maximum SPG and no aortic regurgitation

Mildly mitral valve regurgitation

Severe LV systolic dysfunction

Trivial pulmonary regurgitation

Trivial mitral valve regurgitation

$S \backslash P$ CABG, IHD

RWMA

LV dysfunction $\mathrm{EF}=40 \%$

(B)

Ischemia

Infarction

Ischemia and Infarction

Infarction, likely dilated cardiomyopathy with a low ejection fraction

Ischemia, low ejection fraction at stress

Old infarction

Low ejection fraction at stress, no ischemia no infarction

Left bundle branch block

Ischemic cardiomyopathy

in Figure 2(A), 81 participants were diagnosed cardiac disorders by both techniques, 50 subjects were by eco-cardiograph only, 15 subjects were by scintigraphy only, and 11 subjects did not show any cardiac disorders by both investigations.

When male population was considered (Figure 2(B)), 58 subjects showed cardiac disorders in both diagnostic methodologies; 28 patients were by Echocardiography only; 13 cases were by scintigraphy only, and eight subjects were not diagnosed cardiac disorders by both assessments. When only the female subjects were considered (Figure 2(C)), 23 subjects showed cardiac disorders in both diagnostic methodologies; 22 patients were by Echocardiography only; 2 cases were by scintigraphy only, and three subjects were not diagnosed cardiac disorders by both assessments.

When the study subjects aged less than 50 years were considered (Figure 2(D)), 


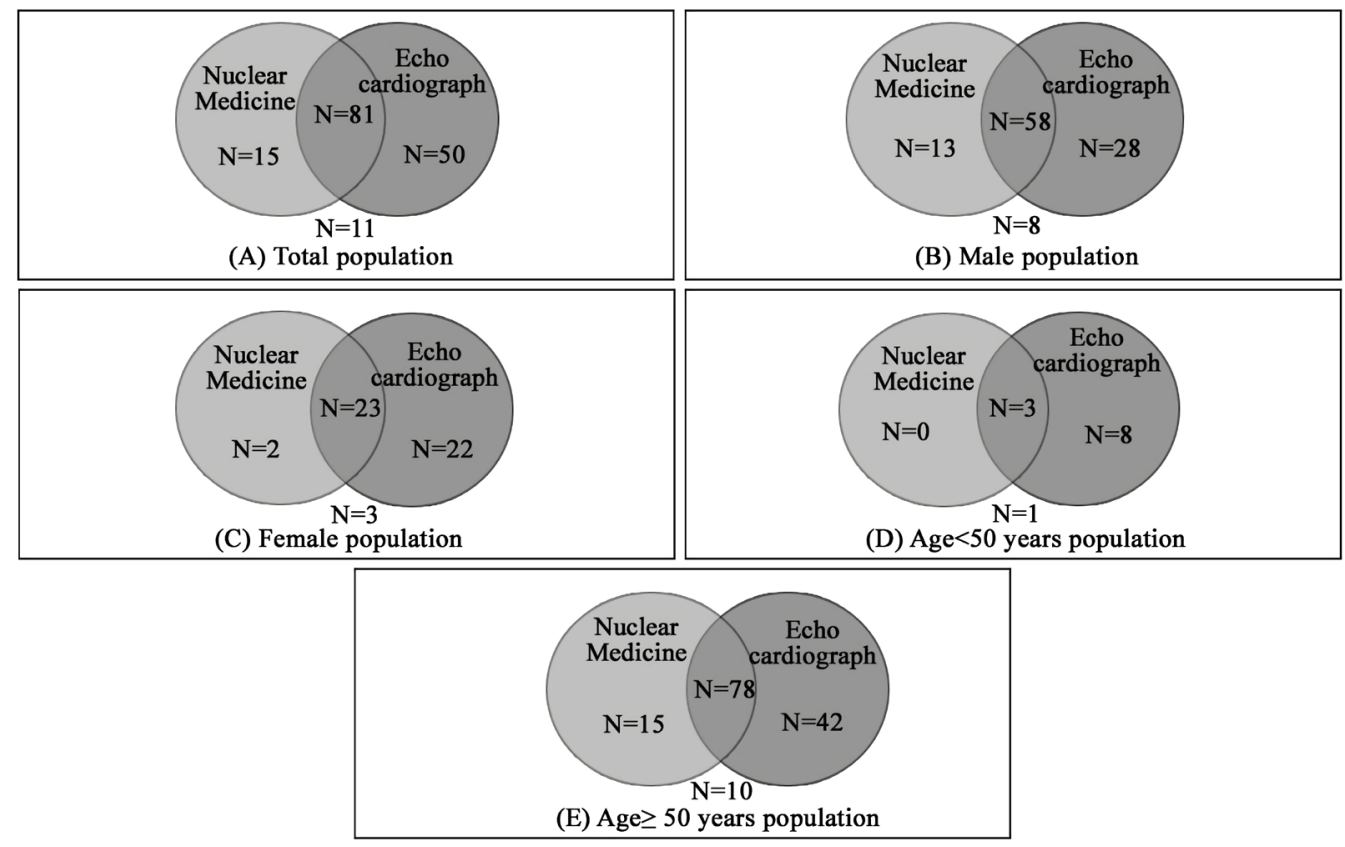

Figure 2. Venn diagrams for diagnosis of cardiac disorder.

three subjects showed cardiac disorders in both diagnostic methodologies; 8 patients were by Echocardiography only; 1 case was by scintigraphy only, and no subjects were found not diagnosed cardiac disorders by both assessments.

When the study, subjects aged more than 50 years were considered (Figure 2(E)), 78 subjects showed cardiac disorders in both diagnostic methodologies; 42 patients were by echocardiography only; 15 cases were by scintigraphy only, and ten subjects were found not diagnosed cardiac disorders by both assessments.

\subsection{Characteristics of Cardiac Scintigraphy}

As shown in Figure 3(A), among the study subjects who are male and less than 50 years age, the prevalence of cardiac disorders by scintigraphy is half in comparison to total participants in this study group.

As shown in Figure 3(B), among the study subjects who are female and less than 50 years age, the prevalence of cardiac disorders by scintigraphy was zero; no case of cardiac disease was detected by scintigraphy. Among the study subjects who are male and more than 50 years age, the prevalence of cardiac disorders by scintigraphy was near around 2.4 times higher in comparison to the number of participants without the cardiac disorder in this study group (Figure 3(C)). As shown in Figure 3(D), among the study subjects who are female and more than 50 years of age, the prevalence of cardiac disorders by scintigraphy was $53 \%$, and the percentage of subjects without cardiac disorder was $46 \%$. Figure 3(E) demonstrated that, among the study subjects who are male, the number of IHD by scintigraphy was almost double without the IHD patient group. Among the study subjects who are female, the number of IHD by scintigraphy was almost the same as that of without IHD (Figure 3(F)). 
(A) Age $<50, \mathrm{M}$

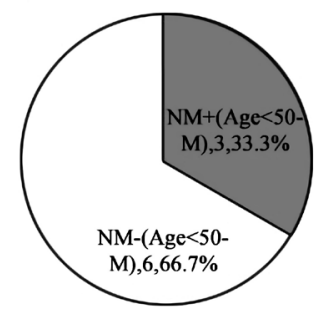

(E) IHD in male

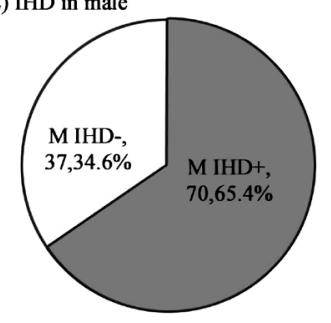

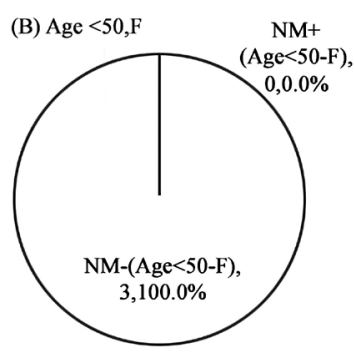

(F) IHD in female

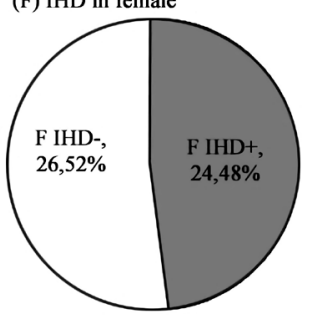

(C) Age $\geq 50, M$

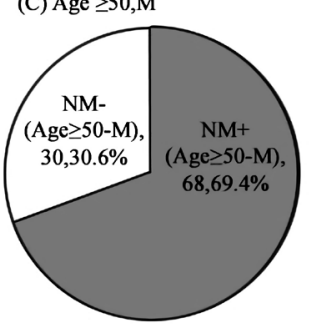

(G) IHD in male (age)

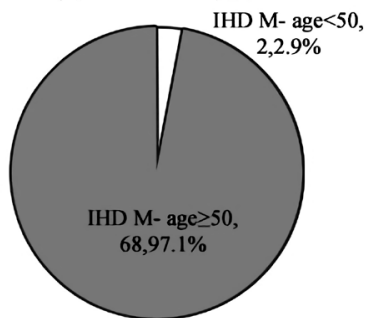

(D) Age $\geq 50, F$

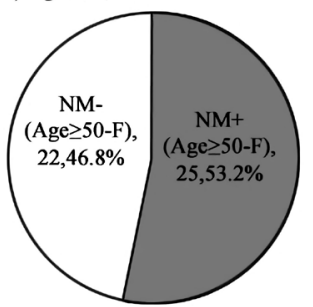

(H) IHD female (age)

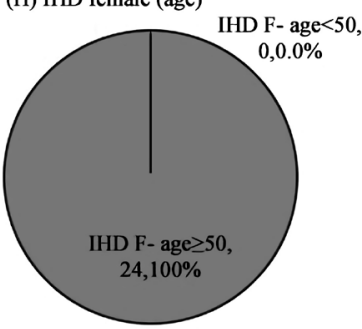

Figure 3. Prevalence of cardiac disorder including IHD diagnosed by nuclear medicine (Scintigraphy).

As shown in Figure 3(G) and Figure 3(H), among both gender study subjects, the IHD patients were almost all who were more than 50 years of age. Comparison by both technologies for ischemic heart diseases (IHD). As shown in Figure 4(A), 36 participants were diagnosed IHD by both technologies, ten subjects were by eco-cardiograph only, 36 subjects were by scintigraphy only, and 53 subjects did not show features of IHD by both investigations. When only male participants were considered (Figure 4(B)), 29 subjects demonstrated by both diagnostic methodologies; 8 patients were by Echocardiography only; 41 cases were by scintigraphy only, and 29 did not have IHD with both technologies.

When only female subjects were considered (Figure $4(C)$ ), seven patients had IHD in both diagnostic methodologies; 2 patients were by Echocardiography only; 17 cases were diagnosed IHD by scintigraphy only; 24 participants had no IHD in either of these investigations.

When the study subjects aged less than 50 years were considered (Figure 4(D)), only one subject had IHD in both technologies; 2 patients were by Echocardiography only; 1 case was by scintigraphy only, and eight subjects were found not diagnosed IHD by both assessments.

In the analysis of the patients more than 50 years of age (Figure 4(E)), 35 subjects showed IHD in both diagnostic methodologies; 8 patients were by Echocardiography only; 57 cases were by scintigraphy only, and 45 subjects were found not diagnosed IHD by both assessments.

Characteristics of Echocardiography

As shown in Figure 5(A), among the study subjects who are male and less than 50 years of age, the prevalence of cardiac disorders by Echocardiography is about eight times higher. In contrast, no echo-negative subject of cardiac disease was found in the same age group of female (Figure 5(B)). Among the study 

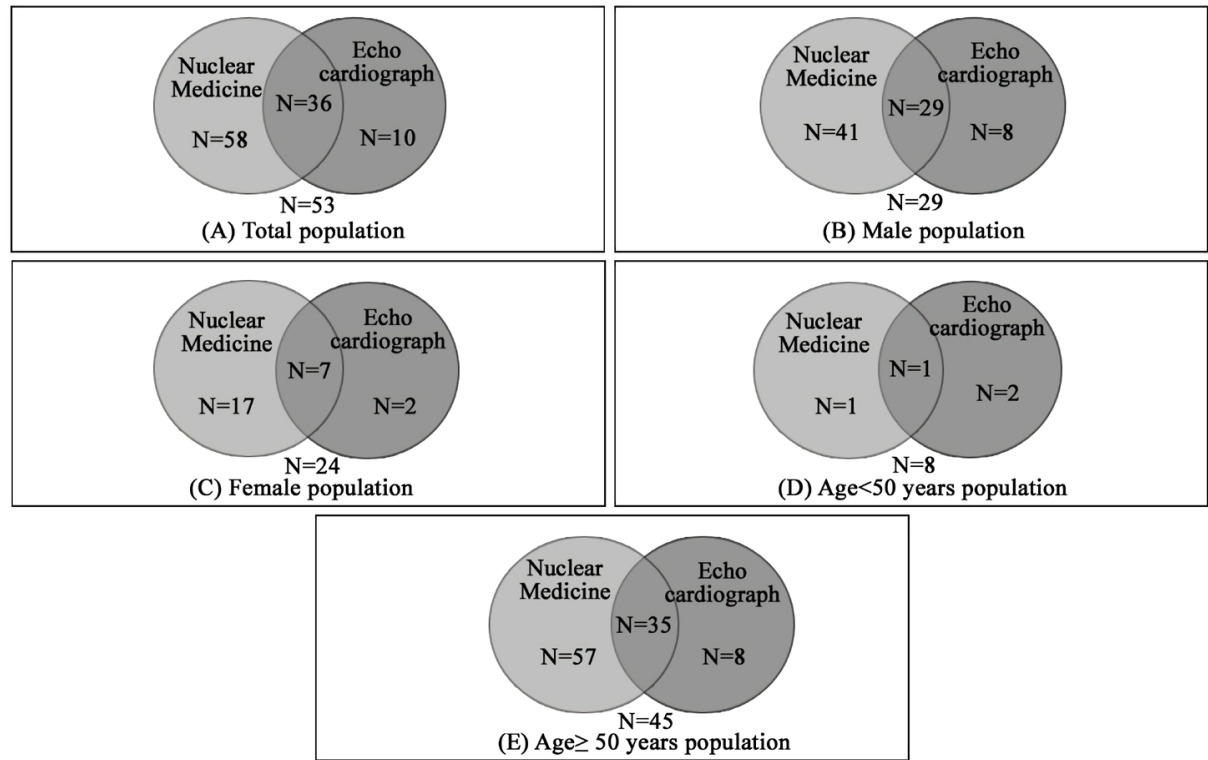

Figure 4. Venn diagrams for ischemic heart disease (IHD).

(A) Age $<50-M$

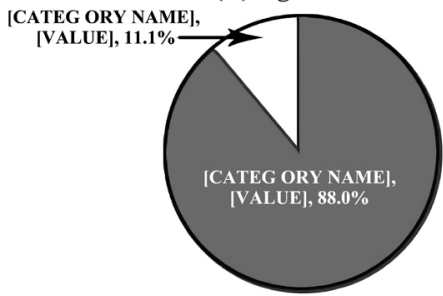

(C) Age $\geq 50-M$

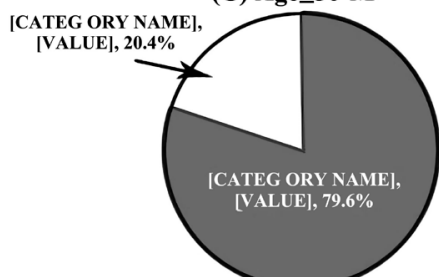

(E) IHD in male

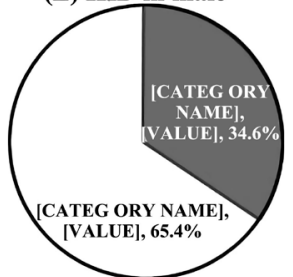

(G) IHD-male (age)

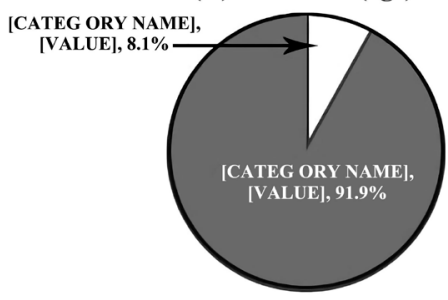

Echo: Echocardiography; IHD: ischemic heart disease; M: male; F: female; +: positive; -: negative
(B) Age $<50-F$

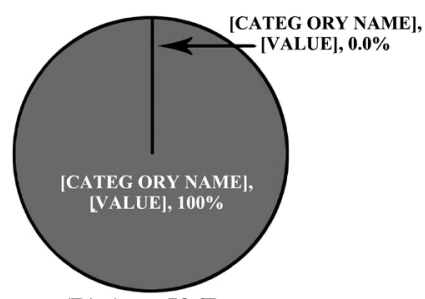

(D) Age $\geq 50-F$

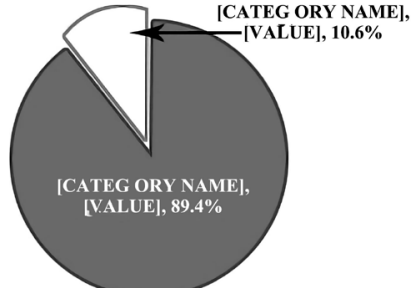

(F) IHD in female

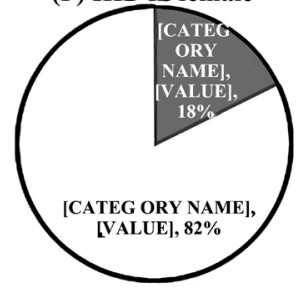

(H) IHD-female (age)

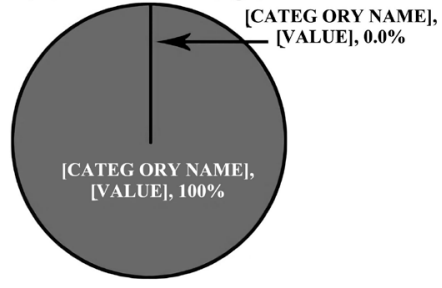

Figure 5. Prevalence of cardiac disorder including IHD diagnosed by Echocardiography. 
subjects who are male and more than 50 years age, the prevalence of cardiac disorders by Echocardiography was 4 times higher in comparison to the number of participants without cardiac disorder in this study group (Figure $5(C)$ ); while in the same age group of female, the prevalence of positive cases of cardiac disorder was about 9 times higher (Figure 5(D)).

Figure 5(E) demonstrated that, among the study subjects who are male, the number of IHD by Echocardiography was near around half in comparison to the number of subjects who were IHD negative. In the female group, the prevalence of IHD positive vs. negative was $18 \%$ vs. $82 \%$ (Figure 5(F)). As shown in Figure 5(G) and Figure 5(H), among both gender study subjects, the IHD patients were almost $90 \%$ to $100 \%$ who were in the age groups more than 50 years of age.

The validity of Echocardiography:

As shown in Table 4, sensitivity was $0.84(0.80-0.89)$; specificity was 0.18 (0.11 - 0.25); PPV: positive predictive value was 0.61 (0.58 - 0.65); NPV: negative predictive value was $0.42(0.26-0.59)$; LR+: likelihood ratio for positive results was 1.02 (0.90 - 1.19); LR-: likelihood ratio for negative results was 0.86 (0.43 1.75) when scintigraphy was considered as reference.

\section{Discussion}

The present study demonstrated the significance and evaluation of cardiac disorders detection by standard Echocardiography and the nuclear medicine scintigraphy at a tertiary levels hospital with the patients having any types of cardiac problems. While conventional Echocardiography detected the greater number of positive cases of cardiac disorders; the percentage of IHD by myocardial scintigraphy was near about two-fold higher than that of Echocardiography. The cardiac disorders were ten-fold more elevated in the patients aged more than 50 years. Also, the cardiac disorders diagnosed by myocardial scintigraphy were almost the patient of IHD.

Transthoracic Echocardiography, a non-invasive (the skin is not pierced) technology, has gained intense popularity as the most standard imaging modality worldwide [5] [7]. In the current study, some of the common types of echocardiography are M-mode echocardiography, Doppler echocardiography, Color Doppler, and 2-D (2-dimensional) Echocardiography was used [20]. It deals

Table 4. Validity of echocardiography considering scintigraphy as reference.

\begin{tabular}{cccc}
\hline Variable & Total number of data $(\mathrm{N}=157)$ & Male $(\mathrm{N}=107)$ & Femail $(\mathrm{N}=50)$ \\
\hline Sensitivity & $0.84(0.80-0.89)$ & $0.81(0.76-0.87)$ & $0.92(0.84-0.97)$ \\
Specifieity & $0.18(0.11-0.25)$ & $0.22(0.12-0.33)$ & $0.12(0.04-0.17)$ \\
PPV & $0.61(0.58-0.65)$ & $0.67(0.63-0.72)$ & $0.51(0.47-0.54)$ \\
NPV & $0.42(0.26-0.59)$ & $0.38(0.21-0.56)$ & $0.60(0.24-0.87)$ \\
LR+ & $1.02(0.90-1.19)$ & $1.05(0.88-1.30)$ & $1.04(0.89-1.18)$ \\
LR- & $0.86(0.43-1.75)$ & $0.82(0.38-1.81)$ & $0.66(0.13-3.16)$ \\
\hline
\end{tabular}


with the information on overall cardiac function, presence of many types of heart disease, follow up the prognosis of heart valve disease over time, evaluation of the effectiveness of medical or surgical treatments and hemodynamics. This technique is mainly useful in diagnosis, assessing prognosis, and determining optimal therapy for several indications, including heart failure, ischemic heart disease, and valve disease [7]. It occupies the top third position in the diagnosis of cardiac disorders next to electrocardiography and chest X-ray [7]. In the present study, more than $83 \%$ of enrolled patients were diagnosed with any types of cardiac disorders by Echocardiography. The rate is quite high, there was no drastic difference in the rate of cardiac disorders in the present study in context to gender, but for IHD, it was about two-fold higher in male than female. In fact, in a significant number of cardiovascular diseases, gender differences are evident in the context of pathophysiology, prevalence rate, clinical presentation, management, and finally, gender-specific outcomes of cardiac disorders (Supplementary Table S1).

Both sex and gender differences are essential in the diagnosis and management of cardiovascular diseases. The expert's panels of institutions and societies from the European Union and the United States have urged and demanded more robust reporting of gender-specific CVD outcomes. In the Euro Heart Survey, Echocardiography was used less frequently in women [21]. Physicians should be informed about this potential bias to reduce it [21]. On the other hand, the male gender is an independent predictor of echocardiographic assessment [22]. Echocardiography also has a potential prediction capability of first cardiovascular events, thus enhances risk stratification for primary prevention in the community [22]. These past study findings are consistent with the current results.

In the present study, most of the study participants were more than 50 years of age; there was no significant difference to be stated in the efficacy of Echocardiography in the detection of cardiac disorders based on age stratification (Supplementary Table S2). IHD has been reported to be developed at a later age for women compared to men, and accordingly, the present study also found that the percentage of cardiac disorders are less in women than men as assessed by Echocardiography (Supplementary Table S3) [23].

MPS is admittedly an integral diagnostic modality and management of CHD. It helps not only in diagnosis but also guides in the decision making of the treatment options. The scintigraphy protocol used in the present study is well unitized [18]. In the current study, the scintigraphy was performed under the supervision of professional guidelines. In the present study, about $61 \%$ of participants were detected cardiac disorders by myocardial scintigraphy with a three-fold higher proportion in the male gender. The total percentage detection rate for cardiac disorders is almost near to that of the percentage of having IHD. This implies that through scintigraphy, the diagnosed cardiac disorders were almost IHD patients. But for echocardiographic assessment, the rate of positivity of cardiac disorders in total and that of IHD are different (83\% vs. 29\%). Al- 
though any comment could not be made on the ability of scintigraphy in the detection of asymptomatic patients in the present study, recent evidence showed that myocardial scintigraphy could also detect asymptomatic ischemia prevailing in early CAD family history and patients with erectile dysfunction, chronic renal failure, and type 2 diabetes. For such case detection, the recommendation to go through scintigraphy investigation is not so popular to date (Supplementary Tables S4-S6) [24].

Through the scintigraphy, the present study could also differentiate the cases of myocardial ischemia, infarction, or having both; whereas, through Echocardiography, this sub-classificationwas not evident in this analysis. The current results again complement the finding that myocardial scintigraphy has a unique diagnostic capability for IHD. Thus, Echocardiography has the potential in diagnosing a wide range of cardiac disorders, whereas scintigraphy is mainly confined to IHD diagnosis, although both of these technologies are already proven beneficial for the detection of CAD. A further recommendation can be made from the present results because myocardial scintigraphy should be performed at a clinical setting in patients with high pretest probability, although even when typical symptoms for ischemia are not evident.

The significant limitations of the study are the lack of data on other potential risk factors and lifestyle factors for heart diseases. The present study included broadly the patients diagnosed with cardiac disorders. Therefore, more stratified analysis based on disease category was not possible in the current study setting. A more sensitive echocardiographic protocol could be used. The data were taken only at the one-time point, therefore difficult to interpret these technologies in the evaluation of treatment modalities and prognostic factors. The current study also lacked biochemical data and data on the presenting feature of cardiac disorders that the participants represented. Besides, the present study did not compare both resting echocardiographic examination and resting nuclear examination to determine which one has higher accuracy in predicting coronary lesions. In general concept, to best determine whether one technique is better than the other, a comparison with a standard gold test such as coronary angiography is necessary to exclude the presence of false positive and false negative examinations in both techniques. Nevertheless, the present study lacked the data from a gold-standard technique for the vital comparison, and this issue must be addressed in future studies. Future studies should also re-evaluate the validity of Echocardiography in the current study setting.

\section{Conclusion}

Both the techniques, namely standard echocardiography and myocardial scintigraphy, are useful in evaluating and detecting cardiac disorders in patients having any type of cardiac problems. While the percentage of cardiac disorders detection capacity is higher in echocardiography than scintigraphy, for diagnosis of IHD, the positive case detection rate is more significant in scintigraphy. Al- 
though the current study setting has limited us to conclude the concrete superiority with statistical standpoint between the technologies used, a more systematic and well-designed study plan awaits the clear conclusion for the study issue investigated here.

\section{Declarations}

\section{Ethics Approval and Consent to Participate}

This study has been reviewed and approved by the Faculty of Applied Medical Sciences, King Abdulaziz University, on 19 September 2019.

\section{Consent for Publication}

Not applicable.

\section{Availability of Data and Materials}

All data generated or analyzed during this study are included in this published article and its supplementary information files.

\section{Funding}

There were no sources of funding.

\section{Conflicts of Interest}

The author declares that they have no competing interests.

\section{Acknowledgements}

The author is very thankful to all the associated personnel and departments in any reference that contributed in/for the purpose of this research.

\section{References}

[1] World Health Organization (2021) Cardiovascular Diseases (CVDs) (Online). https://www.who.int/news-room/fact-sheets/detail/cardiovascular-diseases-(cvds)

[2] Khan, M.A., Hashim, M.J., Mustafa, H., et al. (2020) Global Epidemiology of Ischemic Heart Disease: Results from the Global Burden of Disease Study. Cureus, 12, e9349. https://doi.org/10.7759/cureus.9349

[3] Virani, S.S., Alonso, A., Benjamin, E.J., et al. (2020) Heart Disease and Stroke Statistics-2020 Update: A Report from the American Heart Association External Icon. Circulation, 141, e139-e596.

[4] Naya, M., Murthy, V.L., Blankstein, R., et al. (2011) Quantitative Relationship between the Extent and Morphology of Coronary Atherosclerotic Plaque and Downstream Myocardial Perfusion. Journal of the American College of Cardiology, 58, 1807-1816. https://doi.org/10.1016/j.jacc.2011.06.051

[5] Esmaeilzadeh, M., Parsaee, M. and Maleki, M. (2013) The Role of Echocardiography in Coronary Artery Disease and Acute Myocardial Infarction. The Journal of Tehran University Heart Center, 8, 1-13.

[6] Alizadehasl, A. and Sadeghpour, A. (2018) Echocardiography in Coronary Artery 
Disease and Acute Myocardial Infarction. In: Case-Based Textbook of Echocardiography, Springer, Berlin, 315-321. https://doi.org/10.1007/978-3-319-67691-3_25

[7] Steeds, R.P. (2011) Echocardiography: Frontier Imaging in Cardiology. British Journal of Radiology, 84, S237-S245. https://doi.org/10.1259/bjr/77730594

[8] Mordi, I.R., Badar, A.A., Irving, R.J., et al. (2017) Efficacy of Non-Invasive Cardiac Imaging Tests in Diagnosis and Management of Stable Coronary Artery Disease. Vascular Health and Risk Management, 13, 427-437. https://doi.org/10.2147/VHRM.S106838

[9] Underwood, S.R., Anagnostopoulos, C., Cerqueira, M., et al. (2004) Myocardial Perfusion Scintigraphy: The Evidence. The European Journal of Nuclear Medicine and Molecular Imaging, 31, 261-291. https://doi.org/10.1007/s00259-003-1344-5

[10] Gudmundsson, P., Shahgaldi, K., Winter, R., et al. (2010) Parametric Quantification of Myocardial Ischaemia Using Real-Time Perfusion Adenosine Stress Echocardiography Images, with SPECT as Reference Method. Clinical Physiology and Functional Imaging, 30, 30-42. https://doi.org/10.1111/j.1475-097X.2009.00901.x

[11] Beller, G.A. and Zaret, B.L. (2000) Contributions of Nuclear Cardiology to Diagnosis and Prognosis of Patients with Coronary Artery Disease. Circulation, 101, 1465-1478. https://doi.org/10.1161/01.CIR.101.12.1465

[12] Kazakauskaitè, E., Žaliaduonytė-Pekšienė, D., Rumbinaite, E., et al. (2018) Positron Emission Tomography in the Diagnosis and Management of Coronary Artery Disease. Medicina, 54, 47. https://doi.org/10.3390/medicina54030047

[13] Schinkel, A.F., Bax, J.J., Geleijnse, M.L., et al. (2003) Non-Invasive Evaluation of Ischaemic Heart Disease: Myocardial Perfusion Imaging or Stress Echocardiography? European Heart Journal, 24, 789-800.

https://doi.org/10.1016/S0195-668X(02)00634-6

[14] Chou, R. (2015) Cardiac Screening with Electrocardiography, Stress Echocardiography, or Myocardial Perfusion Imaging: Advice for High-Value Care from the American College of Physicians. Annals of Internal Medicine, 162, 438-447. https://doi.org/10.7326/M14-1225

[15] Fathala, A. (2011) Myocardial Perfusion Scintigraphy: Techniques, Interpretation, Indications and Reporting. The Annals of Saudi Medicine, 31, 625-634. https://doi.org/10.4103/0256-4947.87101

[16] Berman, D.S., Kiat, H.S., Train, K.F., et al. (1994) Myocardial Perfusion Imaging with Technetium-99m-Sestamibi: Comparative Analysis of Available Imaging Protocols Journal of Nuclear Medicine, 35, 683-688.

[17] Kiat, H., Berman, D.S. and Maddahi, J.A. (1993) Myocardial Perfusion Imaging Using Technetium-99m Radiopharmaceuticals. Radiologic Clinics of North America, 31, 795-815.

[18] DePuey, E.G. and Rozanski, A. (1995) Using Gated Technetium-99m-Sestamibi SPECT to Characterize Fixed Myocardial Defects as Infarct or Artifact. Journal of Nuclear Medicine, 36, 952-955.

[19] Lang, R.M., Bierig, M., Devereux, R.B., et al. (2005) Recommendations for Chamber Quantification: A Report from the American Society of Echocardiography's Guidelines and Standards Committee and the Chamber Quantification Writing Group, Developed in Conjunction with the European Association of Echocardiography, a Branch of the European Society of Cardiology. Journal of the American Society of Echocardiography, 18, 1440-1463. https://doi.org/10.1016/j.echo.2005.10.005

[20] Mor-Avi, V., Lang, R.M., Badano, L.P., et al. (2017) Current and Evolving Echocar- 
diographic Techniques for the Quantitative Evaluation of Cardiac Mechanics: ASE/EAE Consensus Statement on Methodology and Indications Endorsed by the Japanese Society of Echocardiography. Journal of the American Society of Echocardiography, 24, 277-313. https://doi.org/10.1016/j.echo.2011.01.015

[21] Cleland, J.G. (2003) Study Group on Diagnosis of the Working Group on Heart Failure of the European Society of Cardiology, the Euro Heart Failure Survey Programme-A Survey on the Quality of Care among Patients with Heart Failure in Europe. European Heart Journal, 24, 442-463.

[22] Tsang, T.S., Barnes, M.E., Gersh, B.J., et al. (2003) Prediction of Risk for First Age-Related Cardiovascular Events in an Elderly Population: The Incremental Value of Echocardiography. Journal of the American College of Cardiology, 42, 1199-1205. https://doi.org/10.1016/S0735-1097(03)00943-4

[23] Aggarwal, N.R., Bond, R.M. and Mieres, J.H. (2018) The Role of Imaging in Women with Ischemic Heart Disease. Clinical Cardiology, 41, 194-202.

https://doi.org/10.1002/clc.22913

[24] Smanio, P.E., Silva, J.H., Holtz, J.V., et al. (2015) Myocardial Scintigraphy in the Evaluation of Cardiac Events in Patients without Typical Symptoms. Arquivos Brasileiros de Cardiologia, 105, 112-122. https://doi.org/10.5935/abc.20150074 


\section{Supplementary Tables}

Table S1. Patient characteristics.

\begin{tabular}{cccc}
\hline Variables & Total $(\mathrm{n}=157)$ & Male $(\mathrm{n}=107)$ & Female $(\mathrm{n}=50)$ \\
\hline Demographics & & & \\
Age, years & $64.0 \pm 0.83$ & $64.3 \pm 1.03$ & $63.5 \pm 1.43$ \\
Age $\geq 50$ years & $145(92.4)$ & $98(91.6)$ & $47(94.0)$ \\
Age $<$ 50 years & $12(7.6)$ & $9(8.4)$ & $3(6.0)$ \\
Echocardiography & & & \\
Echocardiography (positive) & $131(83.4)$ & $86(80.4)$ & $45(90.0)$ \\
IHD (identified by Echo findings) & $46(29.3)$ & $37(34.6)$ & $9(18.0)$ \\
Nuclear medicine & & & \\
Nuclear medicine (positive) & $96(61.1)$ & $71(66.4)$ & $25(50.0)$ \\
Ischemia & $35(22.3)$ & $22(20.6)$ & $13(26.0)$ \\
Infarction & $30(19.1)$ & $26(24.3)$ & $4(8.0)$ \\
Ischemia and Infarction & $29(18.5)$ & $22(20.6)$ & $7(14.0)$ \\
IHD (identified by NM findings) & $94(59.9)$ & $70(65.4)$ & $24(48.0)$ \\
Others & $6(3.8)$ & $4(3.7)$ & $2(4.0)$ \\
\hline
\end{tabular}

${ }^{*} \mathrm{~N}=157$, Values are mean \pm standard error or $\mathrm{n}(\%), \mathrm{IHD}$ : ischemia heart diseases.

Table S2. Validity of the echo cardiograph (gender based).

\begin{tabular}{cccc}
\hline Variable & $\begin{array}{c}\text { Total number of data } \\
(\mathrm{N}=157)\end{array}$ & $\begin{array}{c}\text { Male } \\
(\mathrm{n}=107)\end{array}$ & $\begin{array}{c}\text { Female } \\
(\mathrm{n}=50)\end{array}$ \\
\hline Sensitivity & $0.84(0.80-0.89)$ & $0.81(0.76-0.87)$ & $0.92(0.84-0.97)$ \\
Specificity & $0.18(0.11-0.25)$ & $0.22(0.12-0.33)$ & $0.12(0.04-0.17)$ \\
PPV & $0.61(0.58-0.65)$ & $0.67(0.63-0.72)$ & $0.51(0.47-0.54)$ \\
NPV & $0.42(0.26-0.59)$ & $0.38(0.21-0.56)$ & $0.60(0.24-0.87)$ \\
LR+ & $1.02(0.90-1.19)$ & $1.05(0.88-1.30)$ & $1.04(0.89-1.18)$ \\
LR- & $0.86(0.43-1.75)$ & $0.82(0.38-1.81)$ & $0.66(0.13-3.16)$ \\
\hline
\end{tabular}

*PPV: positive predictive values, NPV: negative predictive values, LR+: likelihood ratio for positive results, LR-: likelihood ratios for negative results, Data are value [95\% confidence interval]; Area under the curve (AUC): $0.5077,95 \%$ CI: $0.4465-0.5689$.

Table S3. Validity of the echo cardiograph (age stratification).

\begin{tabular}{cccc}
\hline Variable & $\begin{array}{c}\text { Total number of data } \\
(\mathrm{N}=157)\end{array}$ & $\begin{array}{c}\text { Age }<50 \text { years } \\
(\mathrm{n}=12)\end{array}$ & $\begin{array}{c}\text { Age } \geq 50 \text { years } \\
(\mathrm{n}=145)\end{array}$ \\
\hline Sensitivity & 0.84 & 1.00 & 0.84 \\
Specificity & 0.18 & 0.11 & 0.19 \\
PPV & 0.61 & 0.27 & 0.65 \\
NPV & 0.42 & 1.00 & 0.40 \\
LR+ & 1.02 & 1.13 & 1.04 \\
LR- & 0.86 & 0.00 & 0.84 \\
\hline
\end{tabular}

${ }^{*}$ PPV: positive predictive values, NPV: negative predictive values, LR+: likelihood ratio for positive results, LR-: likelihood ratio for negative results. 
Table S4. Validity of the echo cardiograph (based on IHD cases).

\begin{tabular}{cccc}
\hline Variable & $\begin{array}{c}\text { Total number of data } \\
(\mathrm{N}=157)\end{array}$ & $\begin{array}{c}\text { Male } \\
(\mathrm{n}=107)\end{array}$ & $\begin{array}{c}\text { Female } \\
(\mathrm{n}=50)\end{array}$ \\
\hline Sensitivity & 0.38 & 0.41 & 0.29 \\
Specificity & 0.84 & 0.78 & 0.92 \\
PPV & 0.78 & 0.78 & 0.78 \\
NPV & 0.48 & 0.41 & 0.59 \\
LR+ & 2.41 & 1.92 & 3.79 \\
LR- & 0.73 & 0.75 & 0.77 \\
\hline
\end{tabular}

${ }^{*} \mathrm{PPV}$ : positive predictive values, NPV: negative predictive values, LR+: likelihood ratio for positive results, LR-: likelihood ratio for negative results, IHD: ischemic heart disease.

Table S5. Validity of the scintigraphy (gender based).

\begin{tabular}{cccc}
\hline Variable & $\begin{array}{c}\text { Total number of data } \\
(\mathrm{N}=157)\end{array}$ & $\begin{array}{c}\text { Male } \\
(\mathrm{n}=107)\end{array}$ & $\begin{array}{c}\text { Female } \\
(\mathrm{n}=50)\end{array}$ \\
\hline Sensitivity & 0.62 & 0.67 & 0.51 \\
Specificity & 0.42 & 0.38 & 0.60 \\
PPV & 0.84 & 0.82 & 0.92 \\
NPV & 0.18 & 0.22 & 0.12 \\
LR+ & 1.07 & 1.09 & 1.28 \\
LR- & 0.90 & 0.85 & 0.81
\end{tabular}

*PPV: positive predictive values, NPV: negative predictive values, LR+: likelihood ratio for positive results, LR-: likelihood ratio for negative results.

Table S6. Validity of the scintigraphy (age stratification).

\begin{tabular}{cccc}
\hline Variable & $\begin{array}{c}\text { Total number of data } \\
(\mathrm{N}=157)\end{array}$ & $\begin{array}{c}\text { Age }<50 \text { years } \\
(\mathrm{n}=12)\end{array}$ & $\begin{array}{c}\text { Age } \geq 50 \text { years } \\
(\mathrm{n}=145)\end{array}$ \\
\hline Sensitivity & 0.62 & 0.27 & 0.65 \\
Specificity & 0.42 & 1.00 & 0.40 \\
PPV & 0.84 & 1.00 & 0.84 \\
NPV & 0.18 & 0.11 & 0.19 \\
LR+ & 1.07 & & 1.08 \\
LR- & 0.90 & 0.73 & 0.88 \\
\hline
\end{tabular}

*PPV: positive predictive values, NPV: negative predictive values, LR+: likelihood ratio for positive results, LR-: likelihood ratio for negative results. 